\title{
Energy management of a building cooling system with thermal storage: a randomized solution with feedforward disturbance compensation
}

\author{
Daniele Ioli, Alessandro Falsone, Maria Prandini
}

\begin{abstract}
We consider a cooling system that comprises a building composed of multiple thermally conditioned zones, a chiller plant, and a thermal storage unit. The electrical energy price is time-varying, and the goal is to minimize the electrical energy cost along some look-ahead time horizon while guaranteeing an appropriate level of comfort for the occupants of the building. To this purpose, we can appropriately set the temperatures profiles in the zones of the building and the cooling energy exchange with the storage. Since the cooling system is affected by stochastic disturbances, we adopt a stochastic formulation of the control problem, where constraints are imposed in probability and measurable disturbances are possibly compensated. The resulting chance-constrained optimization problem is then solved via a randomized approach. Numerical results show a significant reduction of the cost when the feedforward disturbance compensation scheme is adopted.
\end{abstract}

\section{INTRODUCTION}

In this paper we address the optimal energy management of a building cooling system that comprises some thermally conditioned zones in a building, a chiller plant that converts electrical energy in cooling energy conveyed to the zones via a chilled water circuit, and a thermal storage unit.

The goal is to minimize the electrical energy cost paid to the distribution grid utility while satisfying the cooling load request and guaranteeing comfort conditions for the building occupants. To this purpose, we allow the zone temperatures to be modulated within some comfort range and the thermal storage unit to be charged/discharged.

Following [1], we take as control inputs for the zone temperatures modulation their set-points, instead of flows and temperatures of the chilled water circuit as in [2], [3]. This choice highly simplifies the model of the system and is quite sensible if the cooling system has been appropriately dimensioned so as to allow the zone temperatures to track their set-points.

The control inputs (zone temperature set-points and storage contribution) should jointly make the chiller plant operate in highly efficient conditions, and possibly move the electric energy request to the grid to those time slots where prices are lower. The same cost minimization goal was pursued in [1], where we were able to provide a convex formulation of the problem and show that the thermal inertia of the building can be effective as a (passive) thermal storage. Differently from [1], here we account for the

This work is partially supported by the European Commission under the project UnCoVerCPS with grant number 643921.

Daniele Ioli, Alessandro Falsone, and Maria Prandini are with Dipartimento di Elettronica, Informazione e Bioingegneria, Politecnico di Milano, Milano, Italy daniele.ioli, alessandro.falsone, maria.prandini\}epolimi.it stochastic nature of the disturbances acting on the system, e.g., occupancy, outdoor temperature, and solar radiation, in control design, which makes the problem more difficult to solve. In particular, we put in place a (high-level) control layer that tunes the control inputs according to the actual realization of the measurable environmental disturbances. This is achieved by the introduction of a feedforward disturbance compensation scheme. A schematic view of the conceived control system is drawn in Figure 1.

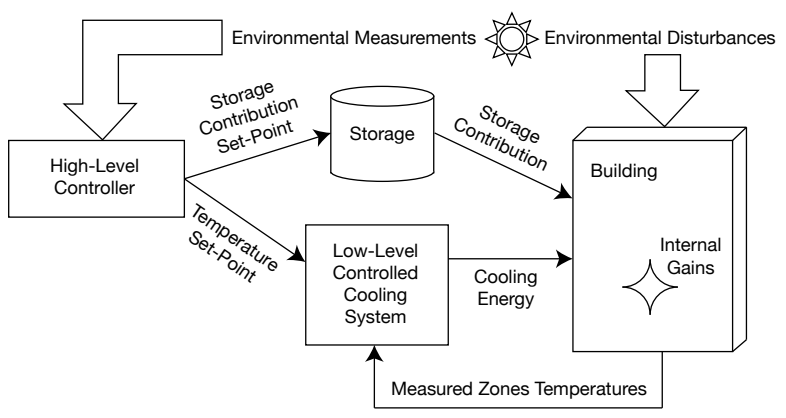

Fig. 1. Proposed energy management scheme with compensation of the environmental disturbances

The presence of uncertainty can be accounted for by adopting either a robust or a probabilistic approach. Although successful in many cases, the robust approach may lead to conservative results, since the stochastic disturbance distribution is neglected when designing the control input, and all disturbance realizations are treated as equally likely. Indeed, it might be the case that low probability disturbance realizations cause a significant deterioration in the cost or even the unfeasibility of some constraint. To overcome these limitations, a problem formulation where a cost function is minimized subject to probabilistic constraints is adopted here. In this setup, a violation of the constraint is accepted, although for few disturbance realizations only, having altogether a probability no greater than a chosen threshold value. This rules out "bad" situations adversely affecting the robust approach. Moreover, probabilistic constraints are the only way to avoid unfeasibility of state constraints when the disturbance has unbounded support (see [4] and the references therein). The dark side of the coin is that probabilistic constraints are in general non-convex and more difficult to treat than standard deterministic constraints. The resulting finite-horizon optimization problem with probabilistic constraints belongs, indeed, to the class of chance-constrained optimization problems, which are known to be hard to solve in general. Convexity of the 
non-stochastic version of the problem in [1] is recovered by adopting a randomized solution to the chance-constrained optimization problem, [5], [6], [7], [8], [9].

We analyze in a numerical example different configurations of the disturbance compensation scheme, where the compensator acts on both the zone temperature set-points and the storage contribution (full compensation), or on the former ones only (partial compensation). The full compensation scheme shows better performance, which is not surprising since it comprises the partial compensation one as a special case.

It is worth mentioning that similar energy management problems have been addressed in the literature mainly via Model Predictive Control (MPC) and stochastic Dynamic Programming (DP) techniques, [10]. Deterministic approaches using MPC and scheduling techniques have been explored, e.g., in [11], [12], [13], [14]. Stochastic approaches based on MPC techniques resting on sampling of possible scenarios are adopted in, e.g., [15], [16]. Stochastic optimal control formulations of the energy management problem can be tackled via DP in certain cases, as shown in [3], [2], [17], [18] where effective solutions to the resulting DP equations have been worked out.

The proposed approach presents some features that distinguish it from the approaches in the literature mentioned above. More specifically, the quantities involved in the optimal management problem are convex as a function of the control inputs; the control inputs include the zones temperatures set-points, which simplifies the enforcement of comfort conditions in the building; stochastic disturbances are explicitly accounted for through a chance-constrained formulation of the problem; a feedforward disturbance compensation scheme is introduced and tuned via a randomized (convex) optimization algorithm. Finally, convexity allows to obtain a randomized solution that is feasible for the original chance-constrained problem.

The rest of the paper is organized as follows. In Section II, we formulate the energy management problem as a chance-constrained optimization program and then provide a randomized solution. Section III presents a numerical example and concludes the paper.

\section{ENERGY MANAGEMENT PROBLEM FORMULATION}

As anticipated in the introduction, we adopt the modeling framework in our previous work [1] for the energy management of a building with a cooling system composed of a chiller plant and a thermal storage. The considered finite time horizon is discretized in $M$ time slot of duration $\Delta t$ to ease the management task.

The building consists of $n_{z}$ zones. Each zone, say zone $j$, has its own temperature set-point, which is defined via a sequence of values $T_{z, j}(k), k=1, \ldots, M$, where $T_{z, j}(k)$ is the temperature enforced at the end of the $k$-th time slot. The state of charge $S$ of the thermal storage evolves according to a simple model $S(k+1)=\alpha S(k)-s(k)$, where $s(k)$ is the cooling energy taken from $(s(k)>0)$ or introduced into $(s(k)<0)$ the storage.
Control inputs can be defined in vectorial form as follows:

$$
\begin{aligned}
& \boldsymbol{T}_{\boldsymbol{z}}=\left[T_{z, 1}(1) \cdots T_{z, n_{z}}(1) \cdots T_{z, 1}(M) \cdots T_{z, n_{z}}(M)\right]^{\top} \\
& \boldsymbol{s}=[s(0) \cdots s(M-1)]^{\top} .
\end{aligned}
$$

Our goal is to appropriately set $\boldsymbol{T}_{\boldsymbol{z}}$ and $s$ so as to minimize the overall electric energy costs while enforcing two kinds of constraints: comfort constraints expressed through bounds on the temperatures, and technical constraints, namely, upper and lower bounds on the thermal energy storage capacity and bounds on the minimum and maximum cooling energy that the chiller can provide.

To this purpose, we start by introducing the cost function

$$
J=\Psi \cdot E_{\ell}\left(T_{z}, s\right)
$$

with $\boldsymbol{\Psi}=[\Psi(1), \ldots, \Psi(M)]$ and $\boldsymbol{E}_{\ell}=\left[E_{\ell}(1), \ldots, E_{\ell}(M)\right]^{\top}$, where $\Psi(k)$ and $E_{\ell}(k)$ are respectively the price of the electricity and the electricity consumed by the chiller in the time slot $k$, when the zone temperature set-points are $\boldsymbol{T}_{\boldsymbol{z}}$ and the energy provided/requested by the storage is $s$.

As detailed in [1], the electricity consumption $E_{\ell}(k)$ can be modeled through the following convex static function of the cooling energy $E_{c h}(k)$ requested to the chiller:

$$
E_{\ell}(k)=c_{1} E_{c h}^{4}(k)+c_{2} E_{c h}^{2}(k)+c_{3} .
$$

In turn, $E_{c h}(k)$ can be computed as

$$
E_{c h}(k)=\sum_{j=1}^{n_{z}} E_{c, j}(k)-s(k),
$$

where $E_{c, j}(k)$ is the energy needed to cool down the $j$-th zone in the building. $E_{c, j}(k)$ can be expressed as the sum of four contributions, namely

$$
E_{c, j}(k)=E_{w, j}(k)+E_{p, j}(k)+E_{\text {int }, j}(k)+E_{z, j}(k),
$$

where $E_{w, j}(k)$ is the heat transferred to zone $j$ from the adjacent walls, $E_{p, j}(k)$ is the heat generated by people that are occupying zone $j, E_{\mathrm{int}, j}(k)$ is the heat generated by electrical equipment, lighting, and radiation through windows, and $E_{z, j}(k)$ is the energy that needs to be exchanged with the zone, characterized by its own thermal capacity, to make its temperature track the set-point.

In [1], it is shown that vector $\boldsymbol{E}_{\boldsymbol{c}}=\left[E_{c, 1}(1) \cdots\right.$ $\left.E_{c, n_{z}}(1) \cdots E_{c, 1}(M) \cdots E_{c, n_{z}}(M)\right]^{\top}$ can be expressed as an affine function of the control input $\boldsymbol{T}_{\boldsymbol{z}}$

$$
\boldsymbol{E}_{\boldsymbol{c}}=A\left(\boldsymbol{d}_{1}, \boldsymbol{d}_{\mathbf{2}}\right) \boldsymbol{T}_{\boldsymbol{z}}+B\left(\boldsymbol{d}_{\mathbf{1}}, \boldsymbol{d}_{\mathbf{2}}\right) .
$$

Matrix $A\left(\boldsymbol{d}_{1}, \boldsymbol{d}_{\mathbf{2}}\right)$ and vector $B\left(\boldsymbol{d}_{\mathbf{1}}, \boldsymbol{d}_{\mathbf{2}}\right)$ have appropriate dimensions and are functions of the disturbance vectors $\boldsymbol{d}_{\mathbf{1}}$ and $\boldsymbol{d}_{\mathbf{2}}$ (see [1] for more details). In particular, $\boldsymbol{d}_{\mathbf{1}}=$ $\left[\begin{array}{llllll}Q_{L W R}(1) & Q_{S W R}(1) & T_{o}(1) & \cdots & Q_{L W R}(M) & Q_{S W R}(M)\end{array}\right.$ $\left.T_{o}(M)\right]^{\top}$ is the vector of measurable disturbances, namely the longwave radiation $Q_{L W R}$, the shortwave radiation $Q_{S W R}$, and the outdoor temperature $T_{o}$, whereas $\boldsymbol{d}_{2}$ collects the other non-measurable disturbances, i.e., occupancy and internal heat generated by appliances.

We implement a feedforward disturbance compensation scheme by allowing the control inputs $T_{z}$ and $s$ to depend 
on the measurable disturbances $\boldsymbol{d}_{\mathbf{1}}$ through the following parametrization:

$$
\begin{aligned}
& T_{z}=\bar{T}_{z}+C_{z} d_{1} \\
& s=\bar{s}+C_{s} d_{1}
\end{aligned}
$$

where $C_{z}$ and $C_{s}$ are lower block-triangular matrices due to control law causality, and $\overline{\boldsymbol{T}}_{z}$ and $\bar{s}$ are further parameters entering affinely.

Comfort and technical constraints that should be satisfied while minimizing the cost function (1) are given by

$$
\begin{array}{ll}
\boldsymbol{T}_{\boldsymbol{z}, \boldsymbol{m i n}} \leq \boldsymbol{T}_{\boldsymbol{z}} \leq \boldsymbol{T}_{\boldsymbol{z}, \boldsymbol{m a x}} & \boldsymbol{E}_{\ell}\left(\boldsymbol{T}_{\boldsymbol{z}}, \boldsymbol{s}\right) \leq E_{\max } \\
\boldsymbol{E}_{\boldsymbol{c}}\left(\boldsymbol{T}_{\boldsymbol{z}}\right) \geq 0 & S_{\min } \leq \boldsymbol{S}(\boldsymbol{s}) \leq S_{\max },
\end{array}
$$

where we made explicit the dependence of $\boldsymbol{E}_{\ell}, \boldsymbol{E}_{\boldsymbol{c}}$ and $\boldsymbol{S}$ on the control inputs $\boldsymbol{T}_{\boldsymbol{z}}$ and $s$, and omitted that on the disturbances $\boldsymbol{d}_{1}$ and $\boldsymbol{d}_{2}$ to simplify the notation.

In the above constraints, $\boldsymbol{T}_{\boldsymbol{z}, \min }$ and $\boldsymbol{T}_{\boldsymbol{z}, \boldsymbol{m a x}}$ are vectors representing temperatures lower/upper bounds in all time slots for all zones, $E_{\max }$ is the maximum amount of electrical energy that can be drawn by the chiller in a time slot, due to its actuation limits, and $S_{\min }$ and $S_{\max }$ are the minimum and maximum capacity of the thermal storage. Vector inequalities should be interpreted component-wise. Further constraints could be added to account for limitations in the low-level controller in charge of the temperature set-point tracking.

Note that both the constraints and the cost function depend on the stochastic disturbance vector $\delta=\left(\boldsymbol{d}_{1}, \boldsymbol{d}_{2}\right)$. A possible strategy to account for uncertainty while avoiding the conservativeness of robust solutions is to minimize the cost and enforce the constraints on a set of realizations of $\delta$ that has a predefined probability $1-\varepsilon$ with $\varepsilon \in(0,1)$ representing the violation probability.

This finally leads to the formulation of the following chance-constrained optimization problem:

$$
\min _{\overline{\boldsymbol{T}}_{\boldsymbol{z}}, C_{z}, \overline{\boldsymbol{s}}, C_{s}, h} h
$$

subject to:

$$
\begin{gathered}
\operatorname{Pr}\left\{\delta: \boldsymbol{\Psi} \cdot \boldsymbol{E}_{\ell}\left(\boldsymbol{T}_{\boldsymbol{z}}, \boldsymbol{s}\right) \leq h, \boldsymbol{T}_{\boldsymbol{z}, \min } \leq \boldsymbol{T}_{\boldsymbol{z}} \leq \boldsymbol{T}_{\boldsymbol{z}, \text { max }},\right. \\
\quad \boldsymbol{E}_{\ell}\left(\boldsymbol{T}_{\boldsymbol{z}}, \boldsymbol{s}\right) \leq E_{\max }, \boldsymbol{E}_{\boldsymbol{c}}\left(\boldsymbol{T}_{\boldsymbol{z}}\right) \geq 0, \\
\left.\quad S_{\min } \leq \boldsymbol{S}(\boldsymbol{s}) \leq S_{\max }\right\} \geq 1-\varepsilon
\end{gathered}
$$

where $\boldsymbol{T}_{\boldsymbol{z}}$ and $s$ are parameterized in terms of $\overline{\boldsymbol{T}}_{\boldsymbol{z}}, C_{z}, \overline{\boldsymbol{s}}$, and $C_{s}$ as in (3) and (4).

Let us collect the optimization variables in vector $\vartheta=$ $\left(\overline{\boldsymbol{T}}_{\boldsymbol{z}}, C_{z}, \overline{\boldsymbol{s}}, C_{s}, h\right) \in \Re^{n_{\vartheta}}$. The (uncertainty dependent) constraint set

$$
\begin{aligned}
\Theta(\delta)=\{ & \vartheta: \boldsymbol{\Psi} \cdot \boldsymbol{E}_{\ell}\left(\boldsymbol{T}_{\boldsymbol{z}}, s\right) \leq h \\
& \wedge \boldsymbol{T}_{\boldsymbol{z}, \min } \leq \boldsymbol{T}_{\boldsymbol{z}} \leq \boldsymbol{T}_{\boldsymbol{z}, \boldsymbol{m a x}} \wedge \boldsymbol{E}_{\ell}\left(\boldsymbol{T}_{\boldsymbol{z}}, \boldsymbol{s}\right) \leq E_{\max } \\
& \left.\wedge \boldsymbol{E}_{\boldsymbol{c}}\left(\boldsymbol{T}_{\boldsymbol{z}}\right) \geq 0, \wedge S_{\min } \leq \boldsymbol{S}(\boldsymbol{s}) \leq S_{\max }\right\}
\end{aligned}
$$

is convex for any $\delta$ uncertainty instance.

With the introduced notations, problem (5) can be seen as an instance of

$$
\begin{aligned}
& \min _{\vartheta \in \Re^{n} \vartheta} V(\vartheta) \\
& \text { subject to: } \operatorname{Pr}\{\delta: \vartheta \in \Theta(\delta)\} \geq 1-\varepsilon
\end{aligned}
$$

where $V(\vartheta)$ is linear as a function of $\vartheta$.

Chance-constrained optimization programs like (6) are difficult to solve and even NP-hard in some cases [19], [20], due to the non-convexity of the probabilistic constraint. One has then to head for an approximate solution. Here, we resort to a randomized method, called the scenario approach, [5], [6], [7]. The idea of the scenario approach is to independently extract $N$ "scenarios" $\delta^{(i)}, i=1, \ldots, N$, of the uncertainty $\delta$ and treat them as if they were the only admissible uncertainty instances. The scenario version of problem (6) then becomes the following (convex) optimization program:

$$
\begin{aligned}
& \min _{\vartheta \in \Re^{n} \vartheta} V(\vartheta) \\
& \text { subject to: } \vartheta \in \bigcap_{i=1}^{N} \Theta\left(\delta^{(i)}\right) .
\end{aligned}
$$

Through disregarding all uncertainty instances but $N$ of them may appear naive, the scenario approach stands on a very solid mathematical ground, as explained next.

Problem (7) is associated to a specific multi-sample $\left\{\delta^{(i)}\right\}_{i=1}^{N}$. Assume that (7) is feasible for any multi-sample. Let $\vartheta_{N}^{\star}$ be its solution. Then, the following theorem holds (see [6] for a proof).

Theorem 1: Select a "confidence parameter" $\beta \in(0,1)$. If $N$ is such that

$$
\sum_{i=0}^{n_{\vartheta}}\left(\begin{array}{c}
N \\
i
\end{array}\right) \varepsilon^{i}(1-\varepsilon)^{N-i} \leq \beta,
$$

where $n_{\vartheta}$ is the number of optimization variables and $\varepsilon$ is the violation parameter, then, $\vartheta_{N}^{\star}$ satisfies $\operatorname{Pr}\left\{\delta: \vartheta_{N}^{\star} \in\right.$ $\Theta(\delta)\} \geq 1-\varepsilon$, with probability no smaller than $1-\beta$.

It is worth noticing that:

- the result in Theorem 1 on the feasibility of the scenario solution for the original chance-constrained optimization problem (6) holds irrespectively of the probability distribution $\operatorname{Pr}$ of the uncertainty vector $\delta$. This entails that disturbances are not required to be independent or uncorrelated;

- the scenario solution does not require one to know the probability distribution $\operatorname{Pr}$ of the uncertainty vector $\delta$ as long as $N$ scenarios $\delta^{(i)}, i=1, \ldots, N$, are available, e.g., as historical data (data-driven approach);

- the explicit bound $N \geq \frac{n_{\vartheta}+1+\ln (1 / \beta)+\sqrt{2\left(n_{\vartheta}+1\right) \ln (1 / \beta)}}{\varepsilon}$ derived in [21] shows that the dependence of $N$ on the confidence parameter $\beta$ is logarithmic. Hence, we can choose a very small value for $\beta$ (like $\beta=10^{-5}$ or even $\beta=10^{-10}$ ) so as to guarantee feasibility beyond any reasonable doubt without affecting $N$ too much;

- the growth of $n_{\vartheta}$ with the time horizon length $M$ may call for a too large number of scenarios and hence, in practice, hamper the a-priori guarantees of the scenario 
solution. This issue may be alleviated by reducing the number of parameters to be tuned via sensible structured parameterizations (see Section III). Also, the possibility of using a receding horizon strategy, where one applies the first calculated value for the control inputs and then recompute, allows to obtain an effective solution based on much fewer scenarios, [15].

\section{NUMERICAL EXAMPLE}

In this section we apply the proposed approach to one of the numerical case studies presented in [1]. We consider a medium-sized office building: $20 \mathrm{~m}$ long, $20 \mathrm{~m}$ wide, and $10 \mathrm{~m}$ tall. The building is divided into three floors, external facades are half glazed and the roof is flat. In particular we focus on the setup which is called "single-zone" in [1], meaning that the temperatures of the three floors have to track the same zone temperature set-point. A 48 hours look-ahead time horizon is discretized in time slots of $\Delta t=10$ minutes length. Differently from [1], we optimize the temperature set-point only every 6 slots (i.e. 1 hour), while the temperature for the slots in between is set by the linear interpolation of two consecutive set-point values. This workaround is actually a nice way to reduce by a factor of 6 the number of optimization variables without loosing the accuracy associated with a finely discretized model. Figure 2 shows the upper and lower bounds for the zone temperature set-point, with stricter bounds during office hours, and the profile of the energy price during the first 24 hours. All the other parameters are set according to those in [1].

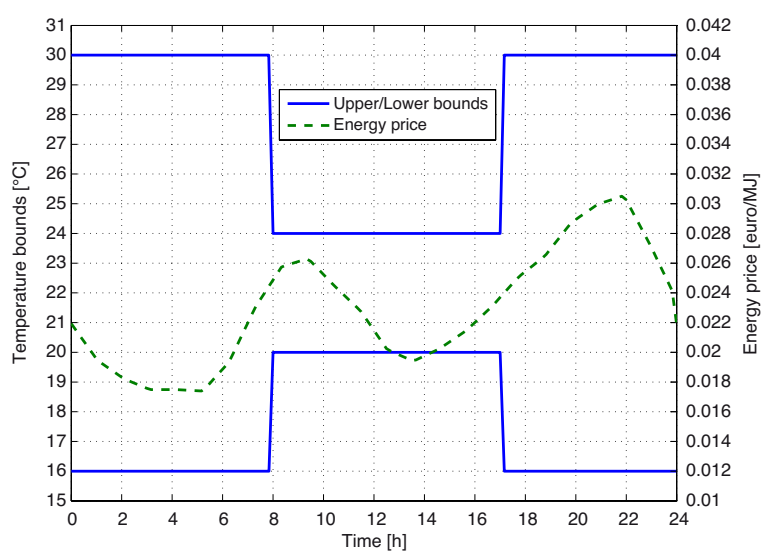

Fig. 2. Temperature bounds (solid line) and energy price (dashed line).

In order to further reduce the number of optimization variables $n_{\vartheta}$ (and, hence, simplify the scenario optimization task), we adopt the following parametrization of the control inputs:

$$
\begin{aligned}
& \boldsymbol{T}_{\boldsymbol{z}}=\overline{\boldsymbol{T}}_{\boldsymbol{z}}+C_{1} \boldsymbol{d}_{1, \boldsymbol{\mu}} \\
& \boldsymbol{s}=\overline{\boldsymbol{s}}+C_{2} \boldsymbol{d}_{1, \boldsymbol{\mu}}+C_{3} Q_{S W R}
\end{aligned}
$$

where $\boldsymbol{d}_{1, \boldsymbol{\mu}}=\left[d_{1, \mu}(1) \ldots d_{1, \mu}(M)\right]^{\top}$ and $d_{1, \mu}(k)$ is given by some weighted average of the measurable disturbances:

$d_{1, \mu}(k)=\mu_{S W R} Q_{S W R}(k)+\mu_{L W R} Q_{L W R}(k)+\mu_{T o} T_{o}(k)$.
Disturbances $Q_{S W R}, Q_{L W R}$, and $T_{o}$ affect the cost function through $\boldsymbol{E}_{\boldsymbol{c}}$. Weights $\mu_{S W R}, \mu_{L W R}$, and $\mu_{T o}$ are then set equal to the linear gains multiplying $Q_{S W R}, Q_{L W R}$, and $T_{o}$ in the affine term $B\left(\boldsymbol{d}_{\mathbf{1}}, \boldsymbol{d}_{\mathbf{2}}\right)$ that appears in equation (2), the idea being that such gains represent a measure of the relevance of the different disturbances. Also, the lower triangular matrices $C_{i}$ are set as follows

$$
C_{i}=\left[\begin{array}{cccc}
c_{i, 1} & 0 & \cdots & 0 \\
c_{i, 2} & c_{i, 2} & \cdots & 0 \\
\vdots & & & \\
c_{i, M} & c_{i, M} & \cdots & c_{i, M}
\end{array}\right]
$$

which further reduces the number of optimization variables. Notice that using structure (10) corresponds in practice to set the control inputs at time $k$ based on the integral of the measurable disturbance realizations up to time $k$, re-scaled by a time-dependent gain $c_{i, k}$. The additional term $C_{3} \boldsymbol{Q}_{S W R}$ contributing to $s$ in (9) allows the storage to compensate fast perturbations related to radiation through windows, while the zone temperature set-point modulation may compensate the effect of environmental disturbances filtered by the slow dynamic of the building structure. The adopted parametrization of the control inputs in (8) and (9) can be recovered as a particular structured case of the more general one in (3) and (4).

Regarding the measurable disturbances $\boldsymbol{d}_{\mathbf{1}}$, we use a set of real data ${ }^{1}$. The available realizations for $\boldsymbol{d}_{\mathbf{1}}$ are only 92 , which is a too low number to provide meaningful a-priori guarantees (low $\varepsilon$ ) via the scenario theory. We then decided to assess a-posteriori the achieved performance by splitting the set of realizations into two subset: 61 used for control input design, and 31 for validation purposes. Accordingly, we generate 92 samples of $\boldsymbol{d}_{\mathbf{2}}$, and use 61 of them for the design phase and the remaining 31 for validation. The occupancy profiles in $\boldsymbol{d}_{2}$ are generated according to the model described in [2], whereas the internal heat realizations in $\boldsymbol{d}_{2}$ are obtained as a function of $\boldsymbol{d}_{1}$ and the occupancy profile (see [1]). Figure 3 represents a possible realization of the disturbances.

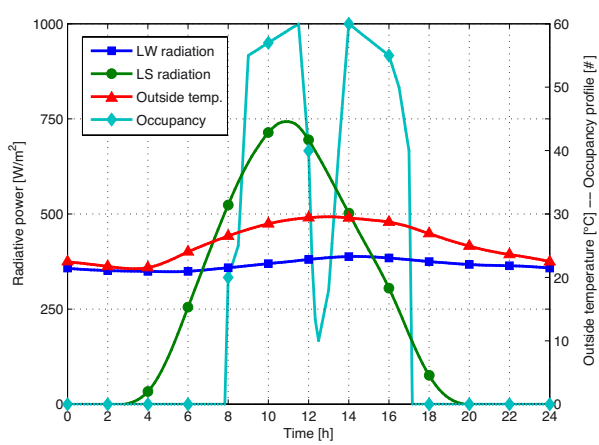

Fig. 3. Realizations of the disturbances.

\footnotetext{
${ }^{1}$ Radiation and meteo data were provided by the "Istituto di Scienze dell'Atmosfera e del Clima (ISAC) - Consiglio Nazionale delle Ricerche", and were taken by a meteorological station operating in Bologna, project 2009/B.04 “Osservatorio BSRN”, National Antartic Research Program.
} 
We next compare three different configurations with/without disturbance compensation and storage.

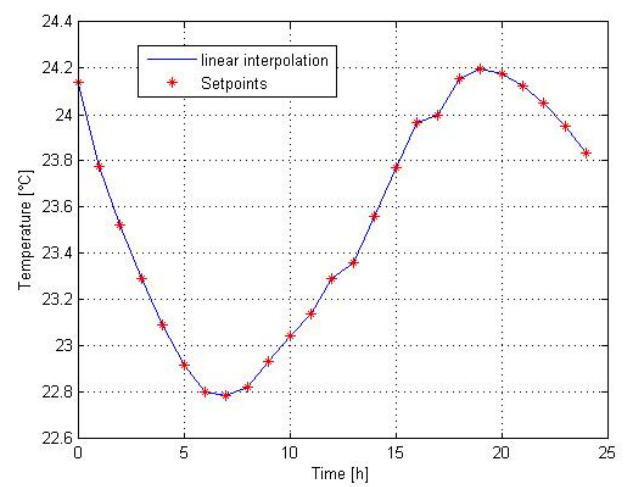

Fig. 4. No compensation: zone temperature set-point.

1) Configuration with no compensation: In this configuration, no disturbance compensation is introduced and the storage is not used. This corresponds to setting $\bar{s}, C_{1}, C_{2}$, and $C_{3}$ equal to zero in (8) and (9). The resulting zone temperature profile $\boldsymbol{T}_{\boldsymbol{z}}=\overline{\boldsymbol{T}}_{\boldsymbol{z}}$ in Figure 4 is characterized by limited fluctuations within the allowed temperature bounds (Figure 2) and, before office hours, a pre-cooling phase during which the temperature is decreased to then exploit the building as a (passive) thermal storage.

2) Configuration with partial compensation: In this configuration, disturbance compensation is added to the zone temperature set-point, while the storage is still not used. This corresponds to setting $\bar{s}, C_{2}$, and $C_{3}$ equal to zero in (9). The zone temperature profiles obtained on validation data show a qualitative behavior that is similar to the profile in Figure 4. Depending on the considered disturbance realization, the pre-cooling phase can be strengthened or weakened. In all tests comfort constraints are never violated, as well as the other technical constraints.

3) Configuration with full compensation and thermal storage: In this last configuration, the storage is used and disturbance compensation is activated on both control inputs. The usage of the storage highly depends on the disturbance realization, which indicates that the compensator tries to apply the best control input for disturbance compensation.

In Figure 5 we report the performance obtained in terms of energy cost for the three different configurations, evaluated on the 31 disturbance realizations kept for validation. As it appears, in almost all validation cases, the presence of the compensator leads to a better performance. The improvement is significant in the case of full compensation with storage, and this is true, in particular, for those realizations corresponding to larger costs. Interestingly, for the full compensation scheme, the costs are weakly varying over all realizations. This is not the case for the other two configurations. In order to better appreciate the compensator performance, we introduce a fictitious "Best" configuration without storage, which is the performance that we could achieve if we knew the actual disturbance realization in

\begin{tabular}{c|cc|cc} 
Best & No compens. & $(\%)$ & Partial compens. & $(\%)$ \\
\hline 18,16 & 24,11 & $(+33)$ & 19,62 & $(+8,03)$ \\
10,68 & 12,93 & $(+21)$ & 12,84 & $(+20,29)$ \\
10,68 & 12,44 & $(+16)$ & 12,75 & $(+19,41)$ \\
13,21 & 17,44 & $(+32)$ & 16,34 & $(+23,66)$ \\
15,58 & 19,48 & $(+25)$ & 16,97 & $(+8,93)$ \\
9,80 & 12,99 & $(+33)$ & 12,56 & $(+28,21)$ \\
20,02 & 27,55 & $(+38)$ & 21,72 & $(+8,50)$ \\
23,33 & 33,15 & $(+42)$ & 24,35 & $(+4,40)$ \\
27,25 & 41,38 & $(+52)$ & 28,24 & $(+3,64)$ \\
26,16 & 36,07 & $(+38)$ & 26,98 & $(+3,15)$ \\
29,40 & 44,30 & $(+51)$ & 30,38 & $(+3,35)$ \\
24,08 & 36,93 & $(+53)$ & 25,14 & $(+4,38)$ \\
31,25 & 45,18 & $(+45)$ & 31,95 & $(+2,25)$ \\
25,33 & 38,07 & $(+50)$ & 26,04 & $(+2,79)$ \\
30,13 & 40,02 & $(+33)$ & 30,60 & $(+1,54)$ \\
21,02 & 27,78 & $(+32)$ & 22,21 & $(+5,66)$ \\
16,14 & 19,16 & $(+19)$ & 17,50 & $(+8,43)$ \\
16,46 & 21,06 & $(+28)$ & 17,80 & $(+8,13)$ \\
21,79 & 25,38 & $(+16)$ & 22,95 & $(+5,35)$ \\
11,90 & 13,31 & $(+12)$ & 13,49 & $(+13,42)$ \\
11,40 & 13,72 & $(+20)$ & 13,68 & $(+20,02)$ \\
13,69 & 15,60 & $(+14)$ & 15,43 & $(+12,66)$ \\
13,44 & 15,74 & $(+17)$ & 15,13 & $(+12,57)$ \\
11,05 & 13,23 & $(+20)$ & 13,28 & $(+20,19)$ \\
8,93 & 11,56 & $(+29)$ & 11,21 & $(+25,57)$ \\
10,34 & 11,73 & $(+13)$ & 12,75 & $(+23,23)$ \\
12,22 & 13,73 & $(+12)$ & 14,07 & $(+15,15)$ \\
8,90 & 12,06 & $(+35)$ & 11,20 & $(+25,82)$ \\
13,12 & 15,69 & $(+20)$ & 14,88 & $(+13,38)$ \\
11,86 & 13,43 & $(+13)$ & 13,52 & $(+14,02)$ \\
16,14 & 18,80 & $(+17)$ & 17,56 & $(+8,81)$
\end{tabular}

TABLE I

COMPARISON BETWEEN BEST, NO COMPENSATION, AND PARTIAL COMPENSATION CONFIGURATIONS (NO THERMAL STORAGE).

advance. We then compare the Best result with the one obtained with the no compensation and partial compensation configurations. Results are presented in Table I. As one can see, the solution without disturbance compensation leads to conservative results, with an increase of the cost up to $53 \%$ with respect to the Best. Adding the disturbance compensator to the zone temperature set-point significantly improves the performance, especially in cases of high cooling load. In Table II the costs of the Best solution are compared with those of a Certainty Equivalence based (CE) solution, where a nominal profile is considered for each disturbance and uncertainty is neglected in the control input design. The CE solution has a worse performance, with an increase in the cost up to $37 \%$. Moreover, in some instances constraints are violated for most of the time horizon (up to $91 \%$ of the overall time). The most commonly violated constraint is that on the cooling energy request to the chiller that cannot be negative in that the chiller cannot provide heating energy. This means that the cooling energy needs are often over-estimated. Uncertainty has to be taken into account to get constraint feasibility for unseen disturbance instances.

\section{REFERENCES}

[1] D. Ioli, A. Falsone, and M. Prandini, "Optimal energy management of a building cooling system with thermal storage: A convex formulation," in ADCHEM, Whistler, British Columbia, Canada, June 2015.

[2] F. Borghesan, R. Vignali, L. Piroddi, and M.Prandini, "Approximate dynamic programming-based control of a building cooling system with 


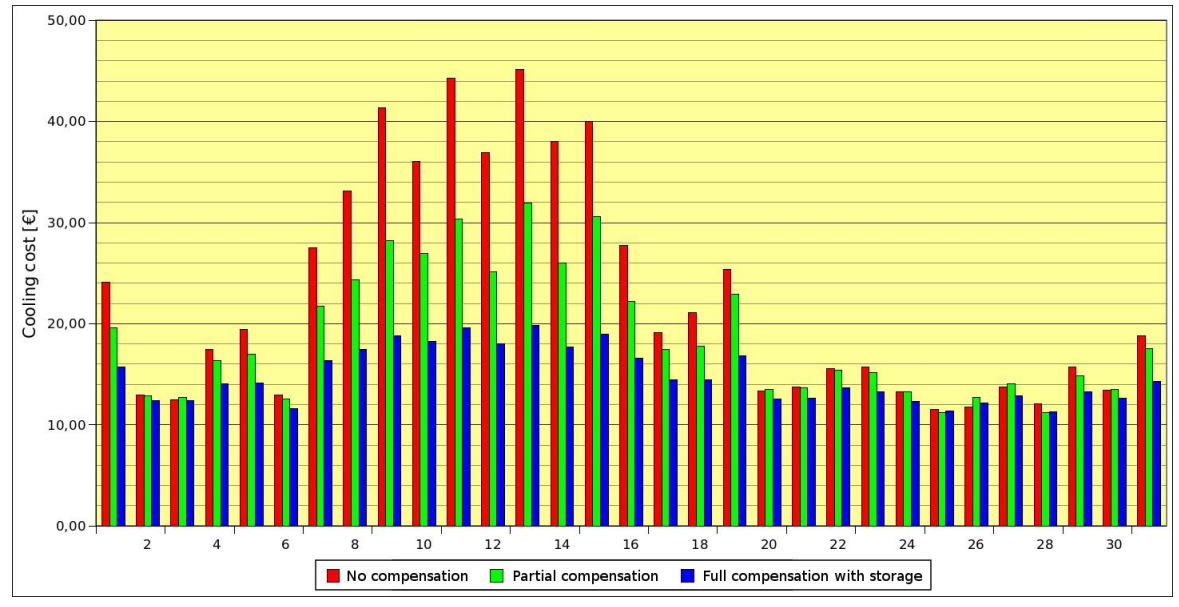

Fig. 5. Bar chart for the comparison of the three proposed configurations.

\begin{tabular}{c|cc|c} 
Best & CE-based & $(\%)$ & Violation $\%$ \\
\hline 18,16 & 21,59 & $(+18,9)$ & 0 \\
10,68 & 12,06 & $(+13,0)$ & 42 \\
10,68 & 11,41 & $(+6,8)$ & 42 \\
13,21 & 15,45 & $(+16,9)$ & 0 \\
15,58 & 17,43 & $(+11,9)$ & 7 \\
9,80 & 11,23 & $(+14,7)$ & 63 \\
20,02 & 24,91 & $(+24,4)$ & 0 \\
23,33 & 29,99 & $(+28,5)$ & 0 \\
27,25 & 37,54 & $(+37,8)$ & 0 \\
26,16 & 33,03 & $(+26,2)$ & 0 \\
29,40 & 40,45 & $(+37,6)$ & 0 \\
24,08 & 33,02 & $(+37,1)$ & 0 \\
31,25 & 41,67 & $(+33,4)$ & 0 \\
25,33 & 34,31 & $(+35,4)$ & 0 \\
30,13 & 37,27 & $(+23,7)$ & 0 \\
21,02 & 25,20 & $(+19,9)$ & 0 \\
16,14 & 17,39 & $(+7,8)$ & 4 \\
16,46 & 18,86 & $(+14,6)$ & 0 \\
21,79 & 23,73 & $(+8,9)$ & 0 \\
11,90 & 12,53 & $(+5,3)$ & 42 \\
11,40 & 12,10 & $(+6,1)$ & 0 \\
13,69 & 14,20 & $(+3,7)$ & 13 \\
13,44 & 14,23 & $(+5,8)$ & 21 \\
11,05 & 11,80 & $(+6,8)$ & 42 \\
8,93 & 10,91 & $(+22,2)$ & 91 \\
10,34 & 10,72 & $(+3,6)$ & 59 \\
12,22 & 12,52 & $(+2,4)$ & 37 \\
8,90 & 11,58 & $(+30,1)$ & 85 \\
13,12 & 13,98 & $(+6,5)$ & 0 \\
11,86 & 12,44 & $(+4,9)$ & 42 \\
16,14 & 17,23 & $(+6,8)$ & 0 \\
& & &
\end{tabular}

TABLE II

COMPARISON BETWEEN THE BEST AND CE-BASED CONFIGURATIONS.

thermal storage," in IEEE ISGT 2013, Copenhagen, Denmark, October 2013.

[3] N. Ceriani, R. Vignali, L. Piroddi, and M. Prandini, "An approximate dynamic programming approach to the energy management of a building cooling system," in ECC, Zurich, Switzerland, July 2013.

[4] L. Deori, S. Garatti, and M. Prandini, "Stochastic constrained control: Trading performance for state constraint feasibility," in Proceedings of the 2013 European Control Conference, 2013, pp. 2740-2745.

[5] G. Calafiore and M. Campi, "Uncertain convex programs: randomized solutions and confidence levels," Mathematical Programming, vol. 102, no. 1, pp. 25-46, 2005.

[6] M. Campi and S. Garatti, "The exact feasibility of randomized solutions of uncertain convex programs," SIAM Journal on Optimization, vol. 19, no. 3, pp. 1211-1230, 2008.

[7] M. Campi, S. Garatti, and M. Prandini, "The scenario approach for

systems and control design," Annual Reviews in Control, vol. 33, no. 2, pp. 149-157, 2009.

[8] R. Tempo, G. Calafiore, and F. Dabbene, Randomized Algorithms for Analysis and Control of Uncertain Systems, with Applications. London, UK: Springer-Verlag, 2013.

[9] M. Prandini, S. Garatti, and J. Lygeros, "A randomized approach to stochastic model predictive control," in 51st IEEE Conference on Decision and Control, Maui, Hawaii, Dec. 2012.

[10] F. Oldewurtel, A. Parisio, C. Jones, D. Gyalistras, M. Gwerder, V. Stauch, B. Lehmann, and M. Morari, "Use of model predictive control and weather forecasts for energy efficient building climate control," Energy and Buildings, vol. 45, pp. 15-27, 2012.

[11] Y. Ma, F. Borrelli, B. Hencey, A. Packard, and S. Bortoff, "Model predictive control of thermal energy storage in building cooling systems," in Proceedings of the $48^{t h}$ IEEE Conference on Decision and Control $/ 28^{\text {th }}$ Chinese Control Conference, Shanghai, P.R. China, Dec. 16-18 2009, pp. 392-397.

[12] Y. Ma, F. Borrelli, B. Hencey, B. Coffey, S. Bengea, and P. Haves, "Model predictive control for the operation of building cooling systems," in American Control Conference, Baltimore (MD), USA, June 30 - July 2 2010, pp. 5106-5111.

[13] M. Behl, T. Nghiem, and R. Mangharam, "Green scheduling for energy-efficient operation of multiple chiller plants," in $33^{\text {rd }}$ IEEE Real-Time Systems Symposium (RTSS), San Juan, Puerto Rico, Dec. 4-7 2012, pp. 195-204.

[14] A. Parisio, E. Rikos, and L. Glielmo, "A model predictive control approach to microgrid operation optimization," IEEE Transactions on Control Systems Technology, vol. 22, no. 5, pp. 1813-1827, Sept 2014.

[15] X. Zhang, G. Schildbach, D. Sturzenegger, and M. Morari, "Scenario-Based MPC for Energy-Efficient Building Climate Control under Weather and Occupancy Uncertainty," in European Control Conference, Zurich, Switzerland, Jul. 2013, pp. 1029-1034.

[16] F. Oldewurtel, C. Jones, A. Parisio, and M. Morari, "Stochastic model predictive control for building climate control," IEEE Transactions on Control Systems Technology, vol. 22, no. 3, pp. 1198-1205, 2014.

[17] F. Borghesan, R. Vignali, L. Piroddi, M. Strelec, and M. Prandini, "Micro-grid energy management: a computational approach based on simulation and approximate discrete abstraction," in $52^{\text {nd }}$ IEEE Conference on Decision and Control, Firenze, Italy, December 10-13 2013.

[18] L. Deori, L. Giulioni, and M. Prandini, "Optimal building climate control: a solution based on nested dynamic programming and randomized optimization," in 53rd IEEE Conference on Decision and Control, Los Angeles, USA, December 2014.

[19] A. Prèkopa, Stochastic Programming. Boston, MA: Kluwer, 1995.

[20] - "Probabilistic programming," in Stochastic Programming, ser. Handbooks in Operations Research and Management Science, A. Ruszczyǹski and A. Shapiro, Eds., vol. 10. London, UK: Elsevier, 2003

[21] T. Alamo, R. Tempo, and A. Luque, "On the sample complexity of randomized approaches to the analysis and design under uncertainty," in ACC2010, Baltimore, MD, USA, Jun. 2010, pp. 4671-4676. 